\title{
Aortic Arch Variants: A Practical Guide to Safe and Timely Catheterization
}

\author{
Maksim Shapiro ${ }^{a} \quad$ Eytan Raz $^{\mathrm{b}}$ Peter Kim Nelson ${ }^{\mathrm{c}}$ \\ aDepartments of Radiology and Neurology, NYU School of Medicine, New York, NY, USA; \\ ${ }^{b}$ Department of Radiology, NYU School of Medicine, New York, NY, USA; ' Departments of \\ Radiology and Neurosurgery, NYU School of Medicine, New York, NY, USA
}

\section{Keywords}

Acute stroke - Angiography · Catheterization - Digital subtraction angiography .

Endovascular procedures - Endovascular treatment - Interventional neuroradiology

\begin{abstract}
Background: Variations in aortic arch anatomy have been extensively described from multiple perspectives including gross anatomy, embryology, associated cardiac and other anomalies, early life presentation, and cross-sectional diagnosis. There is however a paucity of literature with an emphasis on safe and timely catheterization, particularly when the variants are found during emergent or other catheter angiographic procedures without benefit of prior crosssectional vascular imaging. The purpose of this review is to try to fill this gap. Methods: A review of past 1,000 diagnostic and therapeutic catheterizations was performed to identify arch variants, which are presented in order of frequency encountered at our institutions. $\boldsymbol{R e}$ sults: The variants are presented as illustrations and catheter angiographic images, with emphasis on safe and efficient intraprocedural diagnosis and catheterization. Conclusion: Familiarity with more and less common arch variants, along with low threshold for performance of pigtail aortic arch angiography and comfort in use of general purpose and recurved catheters, will ensure success in the vast majority of encountered variations.

(C) 2018 S. Karger AG, Basel
\end{abstract}

\section{Introduction}

Aortic arch variants have been extensively described in the imaging and surgical literature. Many are associated with cardiovascular anomalies or gastroesophageal symptoms (rings and slings) and thus tend to be discovered early in life [1, 2]. Many others however are either isolated or otherwise asymptomatic. These can be found incidentally by astute review of plain film imaging or on cross-sectional studies [3, 4]. Finally, a heretofore unsuspected 


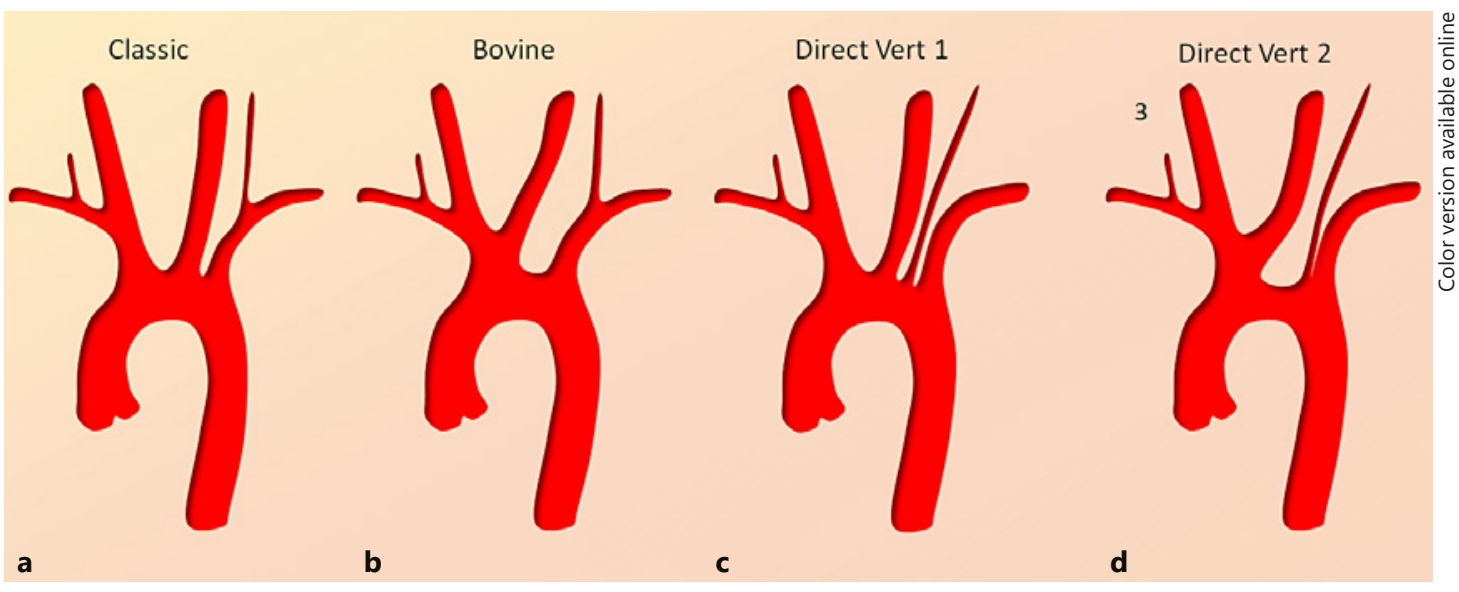

Fig. 1. a-d Common dispositions of the aortic arch. It is important to appreciate that a continuum exists in terms of variation patterns. For example, the right VA can originate in its classic location (a) and anywhere along the more proximal subclavian artery (d) to the fully separate direct origin from the aortic arch (c). The same applies for the "bovine arch" variant (b) where the left CCA can appear to share a common origin with the brachiocephalic artery (truncus bicaroticus) rather than from the brachiocephalic artery itself. ${ }^{\odot}$ neuroangio.org, used with permission here and for all subsequent figures.

arch anomaly may first come to light during elective or emergent endovascular procedure. In this context, it is important to be familiar with the more common variants and to have the endovascular tools and technical expertise at hand to maximize the chances of timely and safe catheterization. The vast majority of arches allow for efficient and safe catheterization once variant anatomy is recognized. Also of practical importance is familiarity with and proficiency in catheterization of typical yet highly technically challenging arches which are frequently encountered in the elderly, particularly in the setting of acute stroke interventions.

While embryology of normal and variant arch development is not discussed in this article, its understanding is very useful, with many sources available to the reader [4-6].

\section{Methods}

An institutional IRB-approved review of our diagnostic and interventional procedure database was performed to identify arch variants. The majority were discovered at the time of catheterization for a variety of diagnostic and therapeutic procedures, with the exception of acute thrombectomy, where aortic arch anatomy is usually known from an immediately preceding CT angiogram. Given the pictorial and technical focus of the paper, demographic and procedural detail data were not collected. A pictorial essay of these variants from the perspective of endovascular management is presented.

Figures 1 and 2 show, in silhouette form, some of the more common (Fig. 1) and less common (Fig. 2) variants and can be used as a guide in a laboratory when a variant is encountered. In many ways, Figure 2 is the more useful one as most practitioners are familiar with dispositions in Figure 1.

\section{Discussion}

\section{Bovine Arch}

This is the most common variant by far and familiar to all endovascular practitioners, with an incidence of about $15-20 \%$ in the literature. A related disposition sometimes termed 


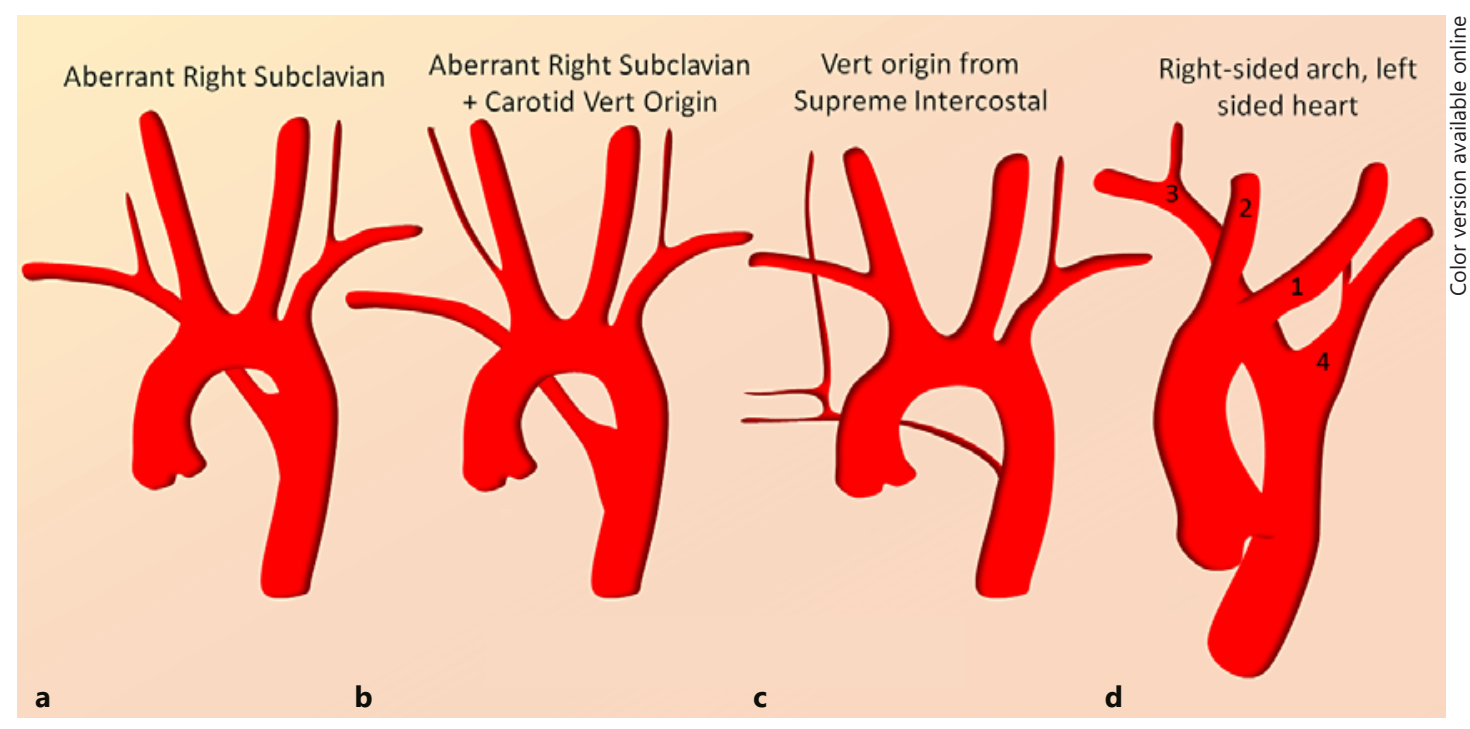

Fig. 2. a-d Less common but in some ways more important variants to be aware of. The most important of these is the right aberrant subclavian artery (a), which is frequently associated with aberrant origin of the right VA from the right CCA (b). Both right and left VA can originate from the proximal descending thoracic aorta distal to the left subclavian artery (c). Some very rare variants such as $\mathbf{d}$ essentially require a pigtail arch aortogram and empiric approach to facilitate catheterization.

"truncus bicaroticus" refers to origin of the left common carotid artery (CCA) from the ostium of the brachiocephalic artery, without a true trunk. The reality of morphologic spectrum between degrees of "bovine arch" and "truncus bicaroticus" contributes to varied estimations of prevalence. In terms of catheterization, familiarity with handling of recurved catheters such as Vitek, Simmons 1, and Simmons 2 is essential. On the other hand, with the increasingly popular radial access, "bovine" left CCA catheterization is often straightforward with a generic hockey-stick type of catheter. Finally, it should be pointed out that "bovine arch" is a misnomer, as the actual arch in a cow has a different disposition [7]; nevertheless, the name stuck.

\section{Direct Left Vertebral Artery Origin from the Aortic Arch}

This is the second most common variant, with an incidence of $~ 4 \%$ [8] in the literature. Again, there is a continuum. Sometimes the left vertebral artery (VA) arises completely separately from the left subclavian ostium (Fig. 1c), while in other cases the origin is either from a common subclavian left vertebral ostium (Fig. 1d) or from the very proximal subclavian artery. Complete hypoplasia of the left VA is quite rare and, if difficulty in finding it is encountered and pathologic occlusion is not confirmed, the two options are either to search for it with a recurved catheter or to perform an arch aortogram. The latter is always our preference, as other still unsuspected variants may be present; furthermore, blind search for a vessel that may or may not exist unnecessarily prolongs the procedure and likely increases procedural hazard. It is important to choose a high enough injection rate to fill the arch and great vessels simultaneously, especially in young patients with normal or high cardiac output which tends to wash the contrast out rather quickly. A $15 \mathrm{~mL} / \mathrm{s}$ for $30 \mathrm{~mL}$ total volume is our standard rate. Breath holding during injection tends to produce more useful images. In a pinch, a usable arch aortogram can frequently be obtained with the diagnostic catheter already in place rather than switching for a pigtail. 

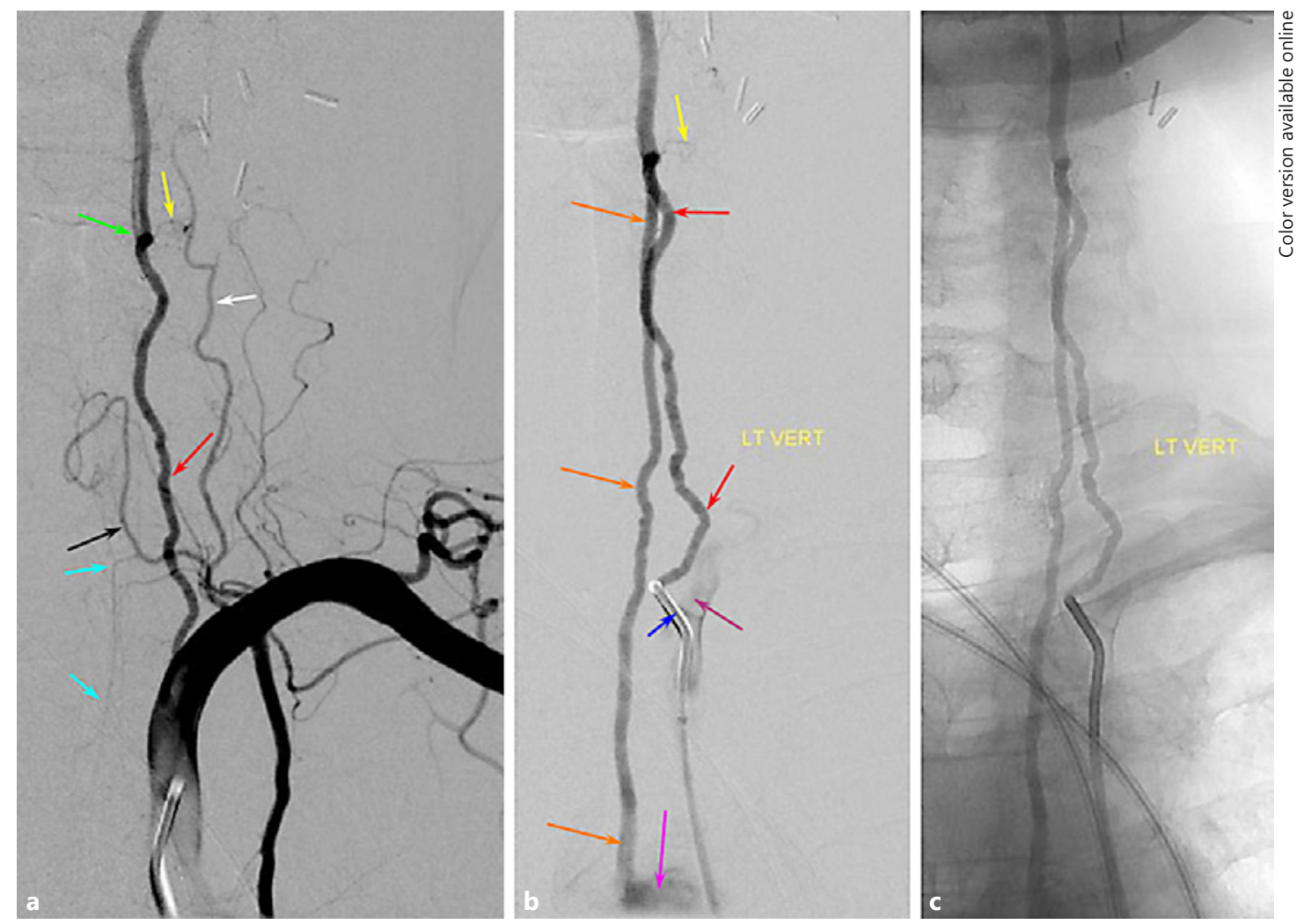

Fig. 3. a-c "Duplicated" VA variant. a Injection of the left subclavian artery is remarkable for presence of unopacified inflow at the C6 vertebral level (green arrow), suggesting that another vessel may be contributing to the supply of the more distal cervical VA. b, c Injections of the proximal subclavian contributor show retrograde opacification of the second, direct aortic arch contributor to the distal VA.

A related variant is the so-called dual-origin left VA (Fig. 3). In this variant two vessels, originating separately from the subclavian artery or from the arch and subclavian artery, join to form a single VA proper in the cervical segment. In this disposition, the more distal "vertebral" artery is usually a branch of the deep cervical artery, for embryologic reasons.

\section{Aberrant Right Subclavian Artery (Fig. 2a)}

This is by far the most common of the "unusual" variants, reportedly in the $0.5-2 \%$ range. The aberrant subclavian artery is the most distal great vessel in this variant (Fig. 4). It is therefore the first vessel to be potentially encountered during femoral catheterization, and often the catheter/wire will spontaneously select the aberrant subclavian artery rather than continuing cranially over the arch. Frequently, the right VA will not be visualized either, arising from the right CCA in aberrant right subclavian dispositions (Fig. 2b, 4, 5). Once this variant is recognized, catheterization of the arch and remaining great vessels is typically no different than in the classic aortic arch disposition. Other anomalies such as bovine trunk can coexist (Fig. 4, 5).

\section{Direct Right VA Origin from the Right CCA (Fig. $2 b, 4,5)$}

Usually this variant is part of the aberrant right subclavian artery disposition; however, it may be seen as an isolated variation also. If difficulty is encountered in locating the origin of the right VA, we typically perform a proximal right brachiocephalic subtraction angiogram, 


\section{Interventional} Neurology

Fig. 4. Aberrant right subclavian artery variant with direct right VA origin from the right CCA (Fig. 2b). This is an example of a "poor man's" flush aortogram performed through a 5-Fr VER general purpose catheter instead of a pigtail. This is sometimes possible, particularly in patients with poor cardiac output, which allows for sufficient slow contrast clearance to opacify the arch and great vessels. Notice the coexisting "bovine" configuration.

\begin{tabular}{l|l}
\hline Intervent Neurol 2018;7:544-555 \\
\hline DOI: 10.1159/000491940 & $\begin{array}{l}\text { @ 2018 S. Karger AG, Basel } \\
\text { www.karger.com/ine }\end{array}$ \\
\hline
\end{tabular}
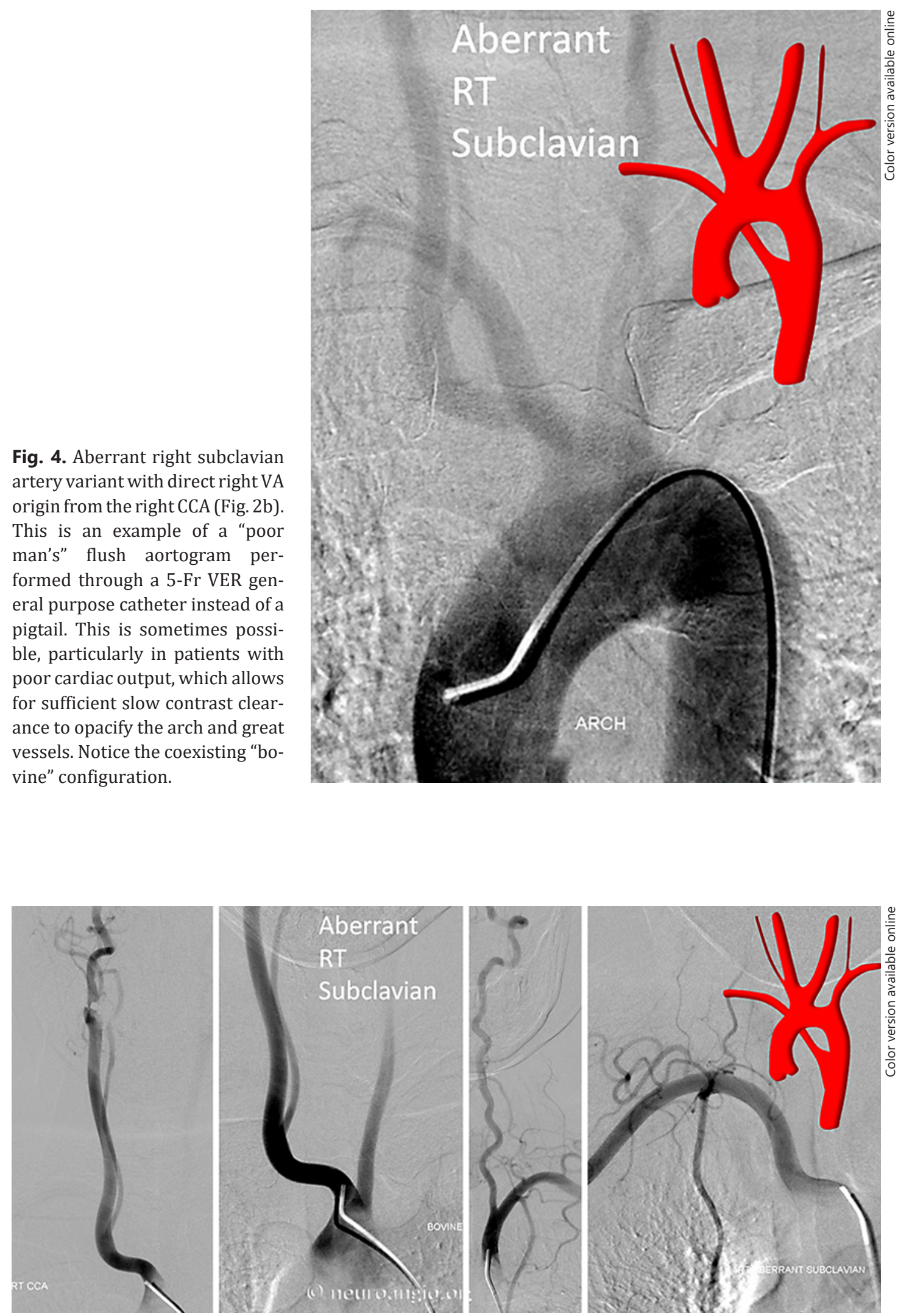

Fig. 5. Dedicated injections of great vessels in the aberrant right subclavian variant whose arch is shown in Figure 4. Note proximal direct right VA origin from the right CCA and bovine configuration. 

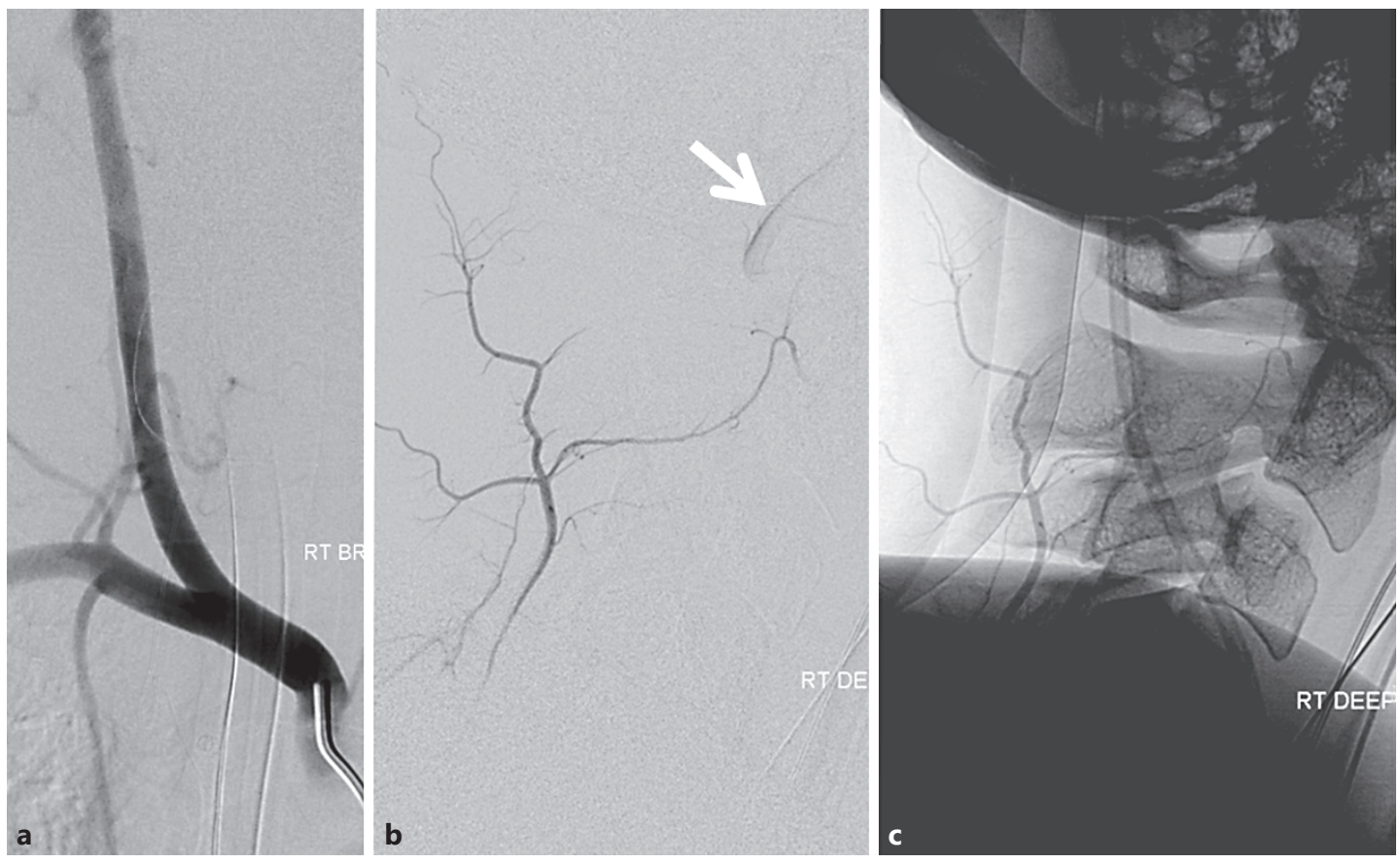

Fig. 6. a Right brachiocephalic injection demonstrating lack of right VA origin. b, c Lateral view injections of the right deep cervical artery demonstrate transient opacification of the right VA (white arrow) via the C2 muscular anastomosis, proving that the right vertebral must be present.

and in most cases the right VA will be found to arise from the right CCA. If there is still uncertainty, arch angiography is the next step. It is important to select a wide craniocaudal view so as to include the thoracic aorta down to about T6 vertebral body, as on very rare occasions the right VA can arise from various portions of the thoracic aorta (see below). Another strategy is to catheterize the left VA and look for unopacified inflow during subtraction angiography to confirm presence of right VA. For an excellent review of the right VA variation see Lemke et al. [8].

\section{Supreme Intercostal Right VA Origin from the Thoracic Aorta (Fig. 2c, 6, 7)}

This is a rare but very important variant to know, otherwise one may be at a loss where to look for the right VA when usual locations are unfruitful. The supreme intercostal artery is the highest (most proximal) segmental artery in the descending aorta. It is responsible for supply of the upper thoracic vertebrae (T1, T2, T3), adjacent tissues, and ribs above the arch. Its longitudinal paravertebral anastomoses are homologous with the deep cervical artery, and indeed the deep cervical and supreme intercostal arteries can be cross-opacified on occasion. Origin of the right VA from the supreme intercostal artery is also related to this homology. A spinal catheter such as RDC is typically used for engaging the ostium of the supreme intercostal artery. Again, if there is uncertainty, an arch aortogram with a field of view down to T6 is very useful when looking for any missing vessel [9].

\section{Persistent Hypoglossal and Proatlantal Variants Type 1 and 2 (Fig. 8, 9)}

While these are not aortic arch variants, they are important variants in the supply of the posterior fossa. They appear as reconstitutions of the VA either from external carotid arteries or CCAs. All represent embryonic variations which are important to understand in their own 
Fig. 7. The same patient as in Figure 6. Right VA origin from the upper thoracic aorta (4-Fr RDC catheter). Notice retrograde opacification of the right deep cervical artery C2 muscular branch (white arrow), the same branch which allowed for opacification of the right VA in Figure $6 \mathrm{~b}$ and $c$.
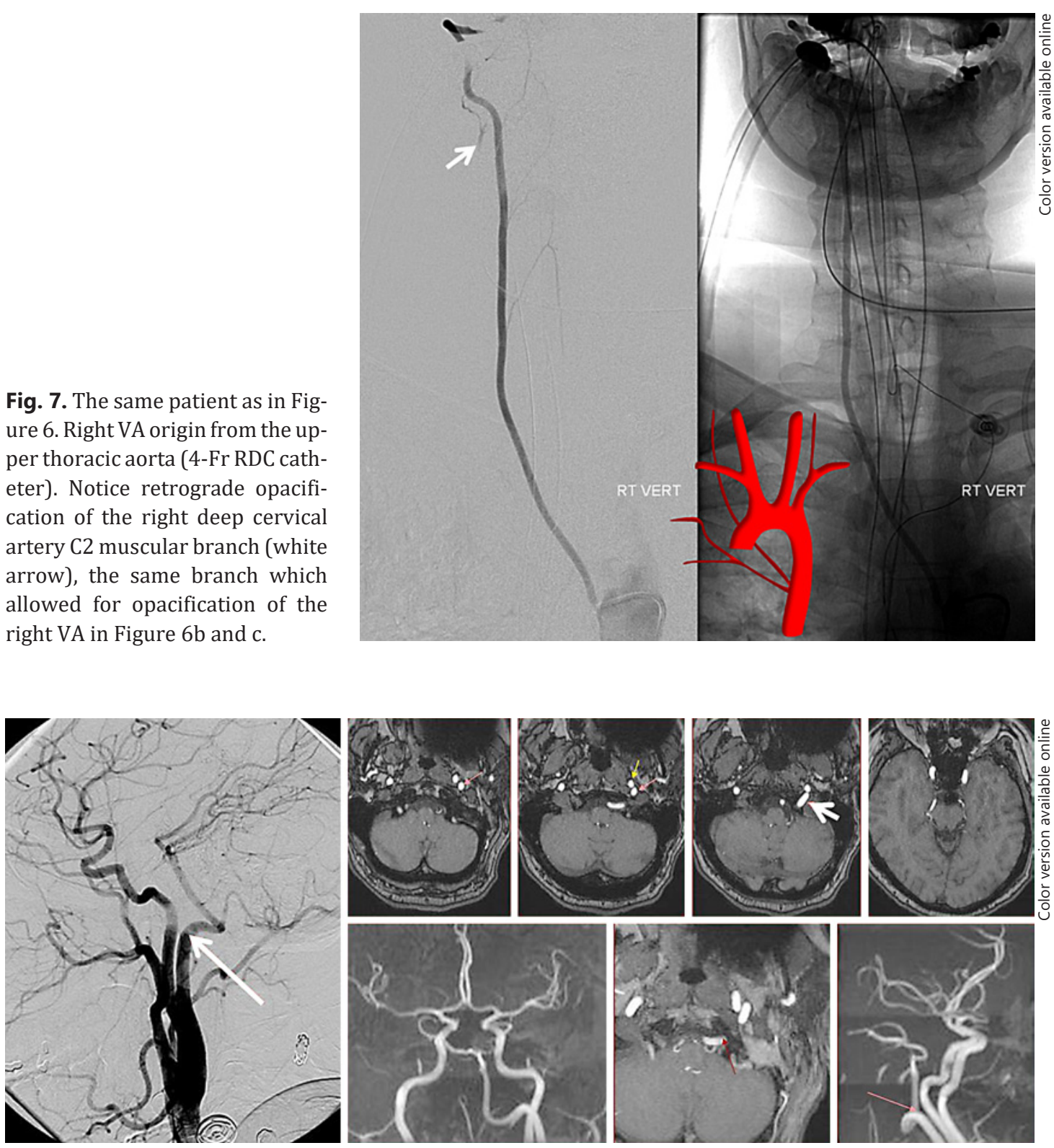

Fig. 8. Persistent hypoglossal artery MRA and lateral angiographic views. The vessel can be readily identified on MRA by its course within the hypoglossal canal (white arrow).

right. A persistent hypoglossal artery is an ascending pharyngeal artery hypoglossal branch anastomosis with the VA. Proatlantal type 1 is an occipital artery to VA anastomosis via the C1 muscular branch (Fig. 9). The proximal VA is usually hypoplastic in such cases, with supply limited to the cervical segment.

\section{Right Arch Left Heart (Fig. 2d, 10)}

This is one of the rarest and most challenging variants from a catheterization standpoint. It can be quite difficult to understand the order in which the vessels originate from the arch on angiography. The usual order, from proximal to distal, is leftCCA, rightCCA, right subclavian artery, left subclavian artery. As in other instances, we suggest a pigtail arch injection (making 


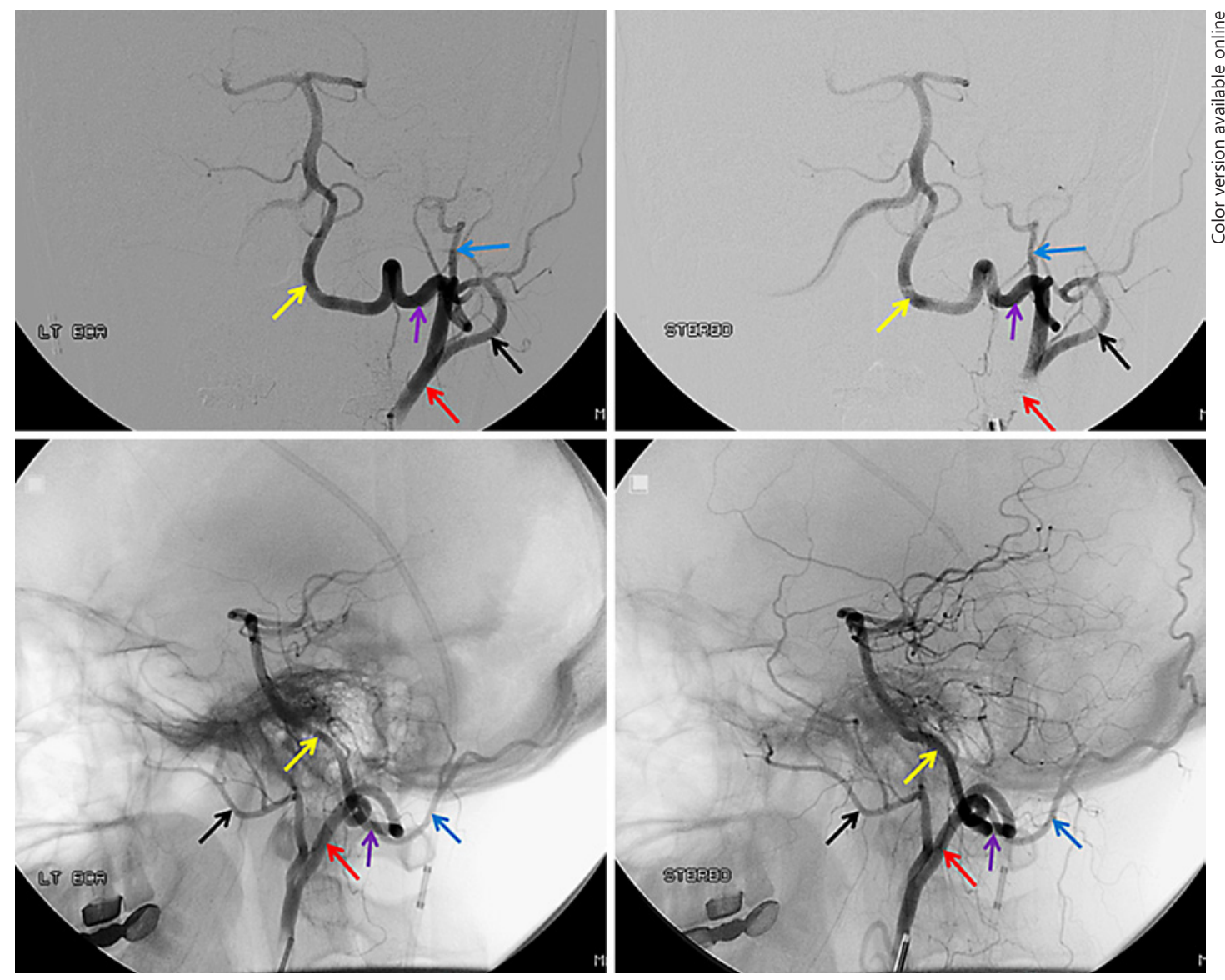

Fig. 9. Type 1 persistent proatlantal artery (red arrow) "arising" from the external carotid artery. In reality the proatlantal artery is the occipital artery (note identical course and lack of separate proximal occipital artery in these views, with the distal occipital artery marked by the blue arrow), with the C1 muscular branch (purple arrow) reconstituting the distal VA (yellow arrow).

sure that the pigtail is sufficiently proximal in the ascending aorta to visualize all vessels). Recurved catheter proficiency is essential.

\section{Right Heart Right Arch (Mirror Images Arch) (Fig. 11)}

This is a self-explanatory mirror image variant mentioned for completeness' sake.

\section{Other Variants}

Many other still rarer variants exist which are not discussed here. While the incidence of different variants ranges in literature, we chose to discuss the ones we had actually encountered, in order of decreasing frequency. The standard approach to these nonstandard situations is to obtain a high-quality pigtail aortogram and take it from there.

Variants of small-branch origin from the great vessels or arch include well-known scenarios such as thyroidea ima or the so-called ascending pharyngeal origin from the internal carotid artery (Fig. 12). See Lo et al. [10] for further information. 
Shapiro et al.: Aortic Arch Variants Practical Guide

Fig. 10. Right arch left heart variant, aortic arch injection.
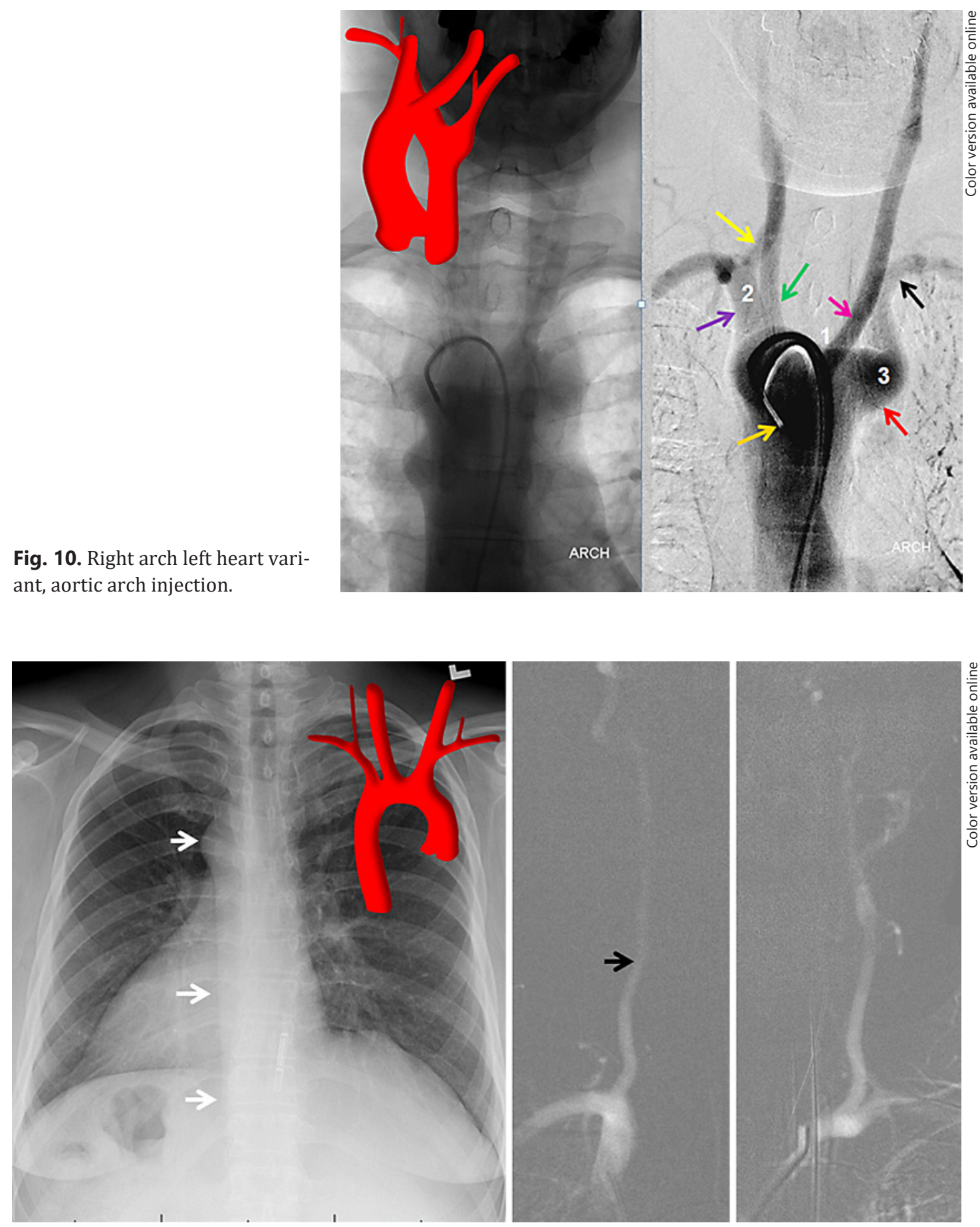

Fig. 11. Mirror image arch (right heart right arch), a self-explanatory variant mentioned for completeness' sake. Notice the outline of the descending aorta on chest X-ray (white arrows). The right VA (black arrow) is typically larger, as expected.

\section{The Challenging "Classic" Arch}

The catheterization challenges in some of these tortuous "candy cane" arches are perhaps greater than in most variant arches, especially once the variant is recognized. The widely used Shuford et al. [4] classification of type 1,2, and 3 arches, based on morphometry of brachio- 

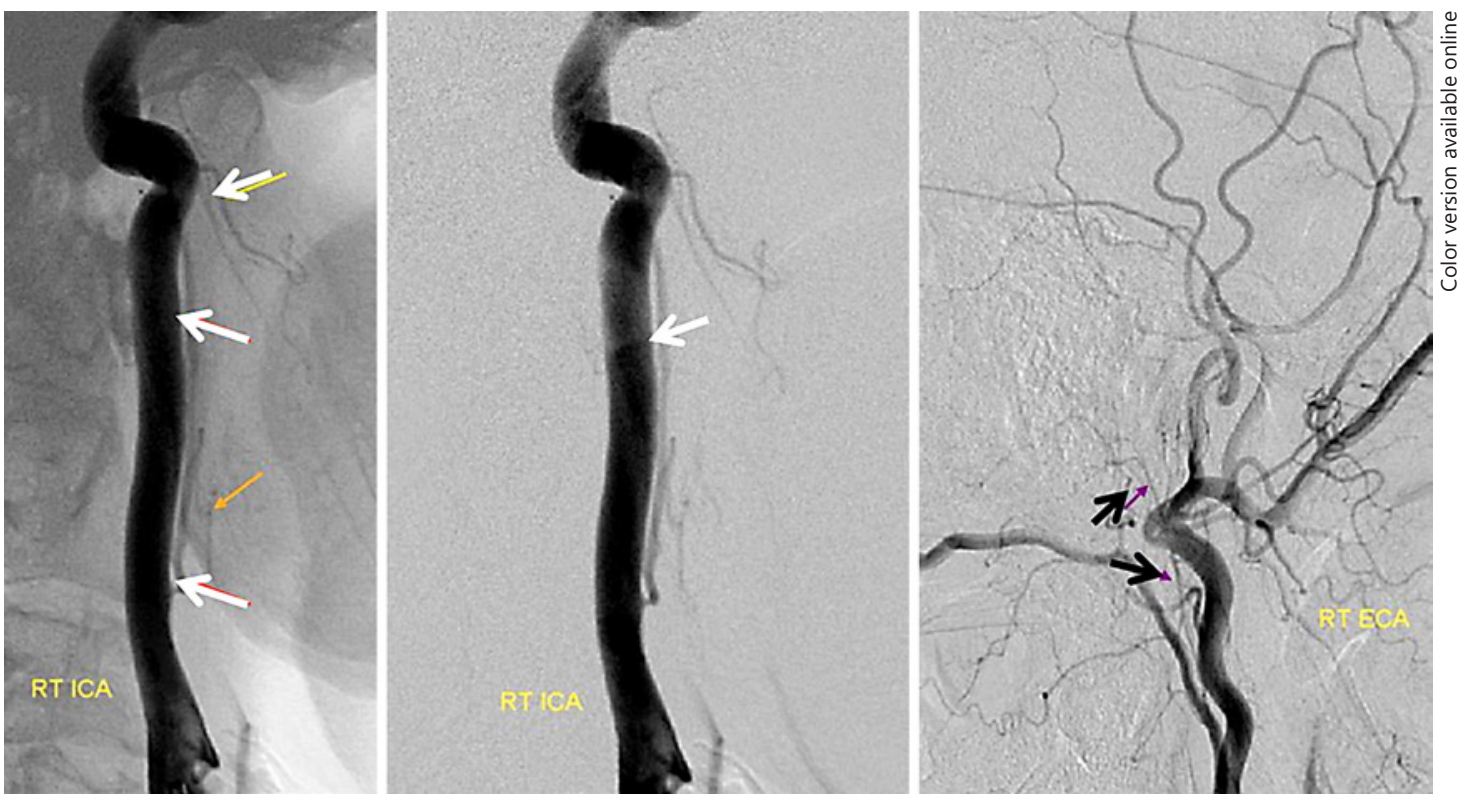

Fig. 12. Right internal carotid artery injection apparently giving rise to pharyngeal division (white arrows) of the ascending pharyngeal artery. The "carotid" trunk is in fact itself part of the ascending pharyngeal artery (aberrant carotid disposition). The neuromeningeal trunk of the ascending pharyngeal artery is marked by black arrows.

cephalic origin relative to arch apex, has important value in standardizing reporting and communication (Fig. 13). In our experience, however, tortuosity of the great vessels themselves (Fig. 13d) is a greater distal catheterization impediment than the arch itself. Certainly operator experience is paramount in these situations and the use of a coaxial access can be very helpful. For example, an 8-Fr 65-cm ARROW-Flex (Teleflex, Ireland) sheath can be very helpful in stabilizing 6-Fr guides such as NeuronMax (Penumbra, Inc., USA), Flexor Shuttle (Cook Medical, USA), or a balloon catheter. A triple-access setup combining the aforementioned long sheaths, guides, and long $(125 \mathrm{~cm})$ diagnostic catheters can be very helpful in catheterizations without need for subsequent exchange maneuvers. On the other hand, familiarity with and availability of several exchange wire types of varied stiffness is essential. Equally important is comfort with alternative access methods such as radial or brachial, which not infrequently will enable a distal enough catheterization with a large enough catheter to allow for intervention.

\section{Radial and Brachial Access Considerations}

Despite extensive cardiology [11] and peripheral catheterization experience, neurodiagnostic and neurointerventional radial access continues to be underutilized [12,13]. Though in our opinion it offers distinct advantages in many settings, most aortic arch variants, with the exception of the bovine arch, are unforgiving to the radial access aficionado, at least from the usual right radial approach. The right subclavian artery in most variants (aberrant right subclavian, right arch left heart) joins the aorta distal to other great vessels and at an unfavorable curve. However, as mentioned above, tortuous "candy cane" arches can sometimes be easier to deal with via radial approach. Our practice is to look at the frequently available chest X-ray for any signs of aberrant anatomy and to prep both groins in addition to the forearm for all radial access cases, for this and other reasons. 


\section{Interventional Neurology}

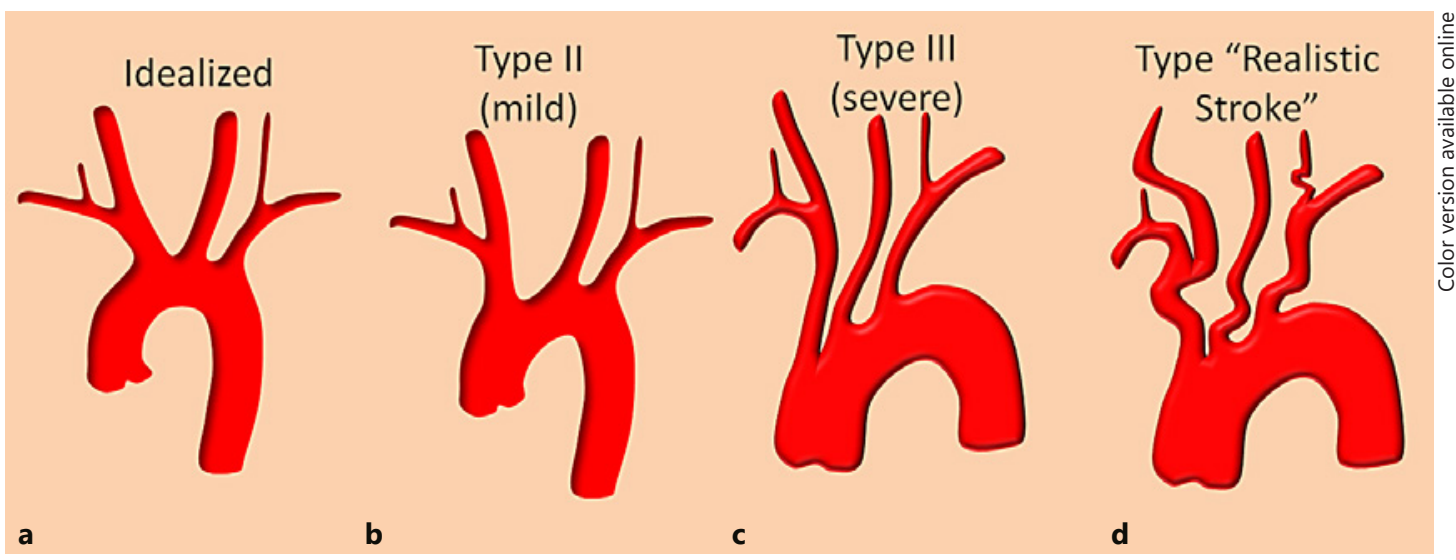

Fig. 13. a-d Well-known arch subtypes related to the tortuosity of the arch itself and perhaps the more realistically encountered combination of coexisting arch and distal great vessel tortuosity (d).

\section{Direct Great Vessel Access}

The frequency, efficacy, and safety of this approach is highly variable and depends in large part on operator experience. Various access and closure methods are highly operatordependent and are beyond the scope of this article.

\section{Study Limitations}

These include a retrospective, nonrandom patient sample leading to potential over- or underrepresentation of the variants. Small incidence of rare variants results in limited experience with catheterization. Our choice of catheterization approach and equipment is necessarily subjective; equally efficacious or better results can be achieved by other methods. A systematic literature review of aortic arch variants was not conducted, and this paper does not discuss the clinical aspects of symptomatic arch variants. All discussion of equipment is quickly rendered obsolete by technical advances.

\section{Future Directions}

Continued refinement of catheterization and imaging equipment will certainly be helpful; however, operator knowledge of anatomy and technical expertise will likely remain cornerstones of procedural success.

\section{Conclusions}

While many arch variants will be discovered clinically or on cross-sectional imaging, the interventional practitioner is sometimes faced with previously unidentified variant arch anatomy in the setting of catheterization. Familiarity with major variants is important in achieving timely and safe access. A low threshold for performance of pigtail aortic arch angiography and proficiency in the use of recurved catheters helps ensure successful catheterization.

\section{Disclosure Statement}

All authors declare no conflict of interest and no financial relationship related to any subjects, devices, or materials discussed in this paper. No financial support was provided by the authors' institution or by any third party for preparation of this paper. 


\section{References}

1 Donnelly LF, Fleck RJ, Pacharn P, Ziegler MA, Fricke BL, Cotton RT: Aberrant subclavian arteries: crosssectional imaging findings in infants and children referred for evaluation of extrinsic airway compression. AJR Am J Roentgenol 2002;178:1269-1274.

2 Ramos-Duran L, Nance JW Jr, Schoepf UJ, Henzler T, Apfaltrer P, Hlavacek AM: Developmental aortic arch anomalies in infants and children assessed with CT angiography. AJR Am J Roentgenol 2012;198:W466W474.

3 Turkvatan A, Buyukbayraktar FG, Olcer T, Cumhur T: Congenital anomalies of the aortic arch: evaluation with the use of multidetector computed tomography. Korean J Radiol 2009;10:176-184.

4 Shuford WH, Sybers RG, Edwards FK: The three types of right aortic arch. Am J Roentgenol Radium Ther Nucl Med 1970;109:67-74.

5 Stojanovska J, Cascade PN, Chong S, Quint LE, Sundaram B: Embryology and imaging review of aortic arch anomalies. J Thorac Imaging 2012;27:73-84.

6 Padget DH: The Development of the Cranial Arteries in the Human Embryo ... With Five Plates, Etc. Contributions to Embryology, 1948, Vol 32. No. 212.

7 Layton KF, Kallmes DF, Cloft HJ, Lindell EP, Cox VS: Bovine aortic arch variant in humans: clarification of a common misnomer. AJNR Am J Neuroradiol 2006;27:1541-1542.

8 Lemke AJ, Benndorf G, Liebig T, Felix R: Anomalous origin of the right vertebral artery: review of the literature and case report of right vertebral artery origin distal to the left subclavian artery. AJNR Am J Neuroradiol 1999;20:1318-1321.

9 Shapiro M: http://neuroangio.org/anatomy-and-variants/aortic-arch/.

10 Lo WW, Solti-Bohman LG, McElveen JT Jr: Aberrant carotid artery: radiologic diagnosis with emphasis on highresolution computed tomography. Radiographics 1985;5:985-993.

11 Kiemeneij F, Laarman GJ, Odekerken D, Slagboom T, van der Wieken R: A randomized comparison of percutaneous transluminal coronary angioplasty by the radial, brachial and femoral approaches: the access study. J Am Coll Cardiol 1997;29:1269-1275.

12 Levy EI, Boulos AS, Fessler RD, Bendok BR, Ringer AJ, Kim SH, Qureshi AI, Guterman LR, Hopkins LN: Transradial cerebral angiography: an alternative route. Neurosurgery 2002;51:335-340; discussion 340-342.

13 Jo KW, Park SM, Kim SD, Kim SR, Baik MW, Kim YW: Is transradial cerebral angiography feasible and safe? A single center's experience. J Korean Neurosurg Soc 2010;47:332-337. 